the respective patients' blood films was remarked on by a technician in the haematology department, who was unaware of a connection between them.

G A Matthews

Department of Haematology,

Hemel Hempstead General Hospital,
Hemel Hempstead, Herts HA2 5 HT

\section{Indomethacin-propranolol interaction}

SIR,-Durao et $a l^{1}$ were first to describe the existence of a drug interaction between indomethacin and propranolol whereby the antihypertensive action of propranolol is attenuated by indomethacin. In a subsequent paper in the $B M \mathcal{A}$ (13 September, p 702) Dr J Watkins and colleagues provide data apparently confirming these findings; however, one aspect of their study design may invalidate their results. The authors state that subjects were given a supply of aluminium hydroxide tablets (375 $\mathrm{mg}$ ) to take as necessary to alleviate gastrointestinal distress caused by indomethacin or the matching placebo. The authors may have overlooked a report by Dobbs et $a l^{2}$ in which the concurrent administration of $30 \mathrm{ml}$ of aluminium hydroxide gel with $80 \mathrm{mg}$ of ora propranolol caused a $58 \%$ decrease in propranolol bioavailability in five human subjects Since subjects in the study by Watkins et a would have been more likely to take aluminium hydroxide tablets during indomethacin administration than during placebo administration, it is quite possible the increase in blood pressure seen during the indomethacinpropranolol period was partially or solely the result of a decrease in propranolol bioavailability. The administration of seemingly unimportant medications such as antacids in a study of this sort must be carefully considered beforehand.

R J MANGIN

Stanford University Medical Center,

Stanford, California

1 Durao V, et al. Lancet 1977;ii:1005-7

- Dobbs JM, et al. Curr Therap Res 1977;21 :887-92.

\section{Antibiotics in surgical treatment of acute} abscesses

SIR,-Mr D H Wilson suggests (18 October, $\mathrm{p}$ 1071) that I did not appreciate that he and his colleagues make a clear distinction between anorectal abscess and fistula-in-ano. I think my lack of this appreciation may reflect my belief that, except when a skin organism is cultured (in preparation), there is no clear distinction between anorectal subsepsis and fistula-in-ano. Any clear distinction can be supported only if there is evidence to show that there is a very low recurrence rate in those abscesses treated by primary suture.

$\mathrm{Mr}$ Wilson quotes three communications ${ }^{1-3}$ in support of primary suture for anorectal sepsis. In the first ${ }^{1}$ the recurrence rate for anal sepsis was $22 \%$ but the mean follow-up was only just over two years and 18 out of 120 patients were also lost to follow-up. The second paper ${ }^{2}$ only included 23 patients with anal sepsis in a total of 150 and there was no mention of recurrence nor follow-up. In the third paper $^{3}$ primary suture was compared with saucerisation and favourable results claimed in relation to comfort, healing time, time off work, and recurrence. The clinical significance of these results is diminished if one remembers that there can now be few surgeons who would saucerise an anorectal abscess when simple linear incision and drainage is all that is necessary; incision and drainage must be obviously accompanied by the search for a fistula. Mr Wilson claims in his last paper that the anal canal was carefully palpated and also examined to demonstrate any internal fistulous opening; unfortunately it is not clear how many were found as 219 patients were accepted into the trial but no mention is made as to the numbers excluded after the finding of a fistula. A total of eight out of 109 patients treated by primary suture developed recurrent sepsis but this figure does not look so impressive when it is realised that only 38 patients were successfully reviewed at one year-that is, the recurrence rate is $8 / 38$ or at least $20.5 \%$.

I would suggest that there is no clear distinction between anorectal sepsis and a fistula and that there is no place for primary suture.

ROGER GRACE

The Royal Hospital,

Wolverhampton WV2 1 BT

1 Wilson DH. Brf Surg 1964;51:828-31.

Jones NAG, Wilson DH. Br J Surg 1976;63:499-501. Goligher JC. Dis Colon Rectum 1976; 19:46-50.
Ger D

\section{Granulocytopenia and septicaemia}

SIR,-Your leading article (25 October, p 1091) on the management of septicaemia in patients with severe granulocytopenia is admirably clear and concise. While briefly mentioning the specific-and growingproblem of infection due to Klebsiella spp you rightly draw attention to the value of cephalosporin therapy. Unfortunately, however, you do not simply refer to this group of antibiotics in general but recommend that whenever ther is a suspicion of Klebsiella infection "cephalothin is indicated." The 1977 publication from the United States which you cite in support undoubtedly reflected the widespread use of the first available cephalosporin which persisted until recently in America. Certainly in Britain this antibiotic had been largely superseded by newer and more satisfactory cephalosporins by $1976 .{ }^{2}$

The drawbacks associated with cephalothin are formidable and include the painful and irritating nature of the injection, which on intravenous use produces thrombophlebitis in a relatively large proportion of patients. The hazard of nephrotoxicity is also significantly greater than with other cephalosporins, apart from cephaloridine; and the simultaneous use of diuretics such as frusemide or of gentamicin and other aminoglycosides potentiates the toxic effect. In addition, cephalothin has poor pharmacokinetic properties, which include vulnerability to metabolic inactivation (by deacetylation), an unusually short serum half life, and moderately high protein binding. As a result comparatively low serum and tissue concentrations are achieved. These deficiencies, together with the high susceptibility of cephalothin to most $\beta$-lactamases (including those of Klebsiella spp), explain why the doses that have to be given are three or four times higher than those needed with other cephalosporins.

The choice of an alternative cephalosporin is now considerably more difficult than it was in 1976, when only five derivatives were available in Britain. Nine are now on the market, and the selection of one of these is all too often made on an arbitrary basis rather than after a full consideration of the varied properties of the different cephalosporins. ${ }^{3}$ The policy in my own hospital is to have available for routine use cephradine, which is the only cephalosporin that can be administered both parenterally and orally. However, as alternatives either cefuroxime or cefoxitin can be ordered if there are bacteriological grounds to support the request.

The likelihood that further (and inevitably more expensive) cephalosporins are to be marketed in the near future makes all the more urgent a wider awareness of the many diverse characteristics of this fascinating group of drugs.

S SELWYN

Westminster Medical School,

London SW1P 2AR

1 Schimpff SC. Med Clin North Am 1977;61:1101-18. 2 Selwyn S. Lancet 1976:ii:616-9. 3 Selwyn S. The beta-lactam antibiotics: penicillins and
cephalosporins in perspective. London: Hodder and cephalosporins in perspective
Stoughton, $1980: 278-98$.

SIR,-Your recent leading article "Granulocytopenia and septicaemia" (25 October, p 1091) requires comment. While most chemotherapists would agree with the substance of the review, the paragraph quoting the work of Love $e t$ al $^{1}$ suggests that in granulocytopenic patients changes in the granulocyte count of as little as $0.1 \times 10^{9} / 1$ may be of importance in determining response to treatment. We would question the validity of this statement on statistical grounds.

In the original paper the median granulocyte count was $0.1 \times 10^{9} / 1$ and neither the method of blood collection (that is, venepuncture or finger stab) nor the technology employed in counting cells was quoted. The method for obtaining granulocyte counts rather than total leucocyte counts was also not stated. These, we feel, are serious omissions, as the statistical errors in the final assessment are dependent on all these factors.

In most routine haematology laboratories in the United Kingdom the statistical errors in measuring such "small" variations in the granulocyte count at this level are so large that accurate interpretation is precluded. While our American counterparts may well have the technology to substantiate their claims this remains to be seen. To most if not all British medical practitioners a change in the granulocyte count of $0.1 \times 10^{9} / 1$ is regarded as being of no significance.

Graham A R Young Alastair G SMITH

University Haematology Department, Western Infirmary,

${ }^{1}$ Love LJ, Schimpff SC, Schiffer CA, Wiernik PH. Am $\mathcal{J}$ Med 1980;68:643-8.

\section{S Lewis and experimental medicine}

SIR,-Dr Brian Livesley in his review of Kenneth Dewhurst's excellent new book about Thomas Willis (18 October, $p$ 1062) takes the opportunity under the title "Four Oxford Men" to devote about $37 \%$ to William Osler and to put C S Lewis on a pedestal. The choice is strange. Osler surprisingly did not consider Willis to be of first rank and relegated him to his bibliotheca secunda. Willis would, I believe, have knocked Lewis off the pedestal. Has Dr Livesley read Lewis's tract savagely attacking vivisection? When I happened to give the 